\title{
Numerical Methods for Compressible Multiphase Flow with Sodium-Water Chemical Reaction*
}

\author{
Akihiro UCHIBORI** and Hiroyuki OHSHIMA** \\ **Japan Atomic Energy Agency, \\ 4002 Narita-cho, Oarai-machi, Higashi-Ibaraki-gun, Ibaraki 311-1393, Japan \\ E-mail: uchibori.akihiro@jaea.go.jp
}

\begin{abstract}
In order to establish a safety evaluation method of a steam generator of sodium-cooled fast reactors, a computer program called SERAPHIM calculating compressible multicomponent multiphase flow with sodium-water chemical reaction under tube failure accident has been developed. In this study, a numerical model for liquid droplet entrainment from an interface of the gaseous jet and its transport was newly constructed to evaluate the environment of the liquid droplet impingement erosion. The applicability of the SERAPHIM program which incorporates the droplet entrainment / transport model was investigated through the analysis of vertical discharging of water vapor in the liquid sodium pool under the actual condition of the steam generator. The analysis reproduced appearance of the underexpanded vapor jet. The calculated peak temperature agreed with the experimental results well. Also, appearance of the dispersed phase of droplets in the reacting jet and its acceleration by the supersonic gaseous flow were represented successfully.
\end{abstract}

Key words: Sodium Cooled Fast Reactor, Steam Generator, Sodium Water Reaction, Liquid droplet Entrainment, Numerical Analysis

\section{Introduction}

In a steam generator ( $\mathrm{SG}$ ) of sodium-cooled fast reactors, heat exchange takes place between liquid sodium in a shell side and water or vapor inside a heat transfer tube. Liquid sodium as a coolant has an excellent heat-transfer characteristic. On the other hand, it has a chemical reactivity with oxygen and water. When pressurized water or vapor leaks from a failed heat transfer tube, a high-velocity, high-temperature and corrosive jet with sodium-water chemical reaction is formed in the shell side (see Fig. 1). It is known that the reacting jet may cause wastage on adjacent tubes. The wastage is attributed to erosion, flow accelerated corrosion (FAC) or combination of them. Significant progress of the wastage causes a secondary failure (failure propagation). There is also a possibility that degradation of a mechanical strength from a rise of the tube wall temperature may cause over-heating rupture. The failure propagation will lead to expansion of the damage, exchange of the equipment and long-term shutdown of the plant. Prevention of the failure propagation is a major concern in design of the SG of a commercial fast reactor.

Numerical analysis is an effective way to evaluate possibility of the occurrence of failure propagation. It has a great advantage over mock-up test in aspects of a cost, development period and flexibility for the change of the design. To enable safety assessment of the SG from numerical analysis and minimal mock-up test, we are developing a new 


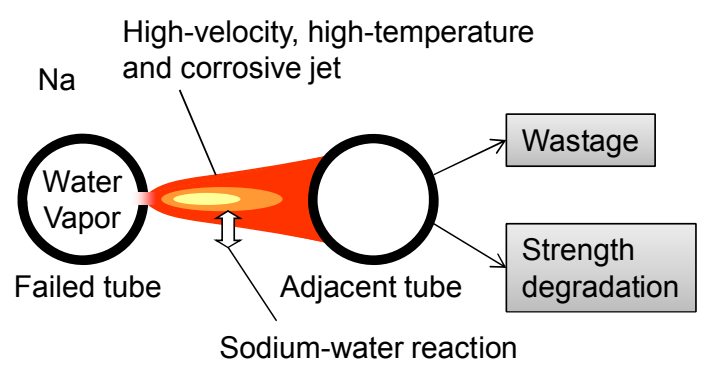

Fig. 1 Reacting Jet under Tube Failure Accident

mechanistic evaluation system for the tube failure accident. The evaluation system consists of the multiple computer programs. As one component of the system, there is a computer program called SERAPHIM (ㅇoium-watEr Reaction Analysis : $\underline{\text { PHysics of }}$ Interdisciplinary Multi-phase flow) calculating compressible multicomponent multiphase flow with sodium-water chemical reaction ${ }^{(1-3)}$. The SERAPHIM program predicts profiles of the velocity, temperature and concentration, which are necessary to evaluate possibility of the failure propagation. The SERAPHIM program is based on the mechanistic models. A multi-fluid model considering compressibility was adopted to calculate the multiphase flow with water, liquid sodium and multicomponent gas. Mechanisms of the sodium-water chemical reaction were investigated and a new mechanistic model was constructed in the previous studies.

Liquid droplet impingement erosion (LDIE) is thought to be one of the main causes of the wastage on the adjacent tubes. In this study, to evaluate the environment of the LDIE, the numerical models for droplet entrainment from the gas-liquid interface and its transport process was newly constructed and incorporated into the SERAPHIM program. The purpose of this paper is to demonstrate applicability of the existing and the newly constructed numerical models.

Water vapor going out from an opening of the tube becomes a supersonic jet because a sodium-side pressure is lower than a critical pressure of water vapor inside the tube. Validation for a gaseous supersonic jet is one of the key issues in development of the SERAPHIM program. The numerical analysis of the three-dimensional underexpanded air jet in the air is firstly presented to confirm adequacy of the governing equations and the solution method considering compressibility. Kuehner et al. ${ }^{(4)}$ and Woodmansee et al. ${ }^{(5)}$ measured the profile of the pressure along the centerline of the underexpanded air jet in the air by using the coherent anti-Stokes Raman scattering (CARS) technique. The calculated pressure was compared to the experimental data. Kudo et al. ${ }^{(6)}$ carried out the experiment on the argon gas jet in the water pool and measured the void fraction around the rod installed above the gas inlet nozzle. Their experiment was chosen as one of the validation problem for the multiphase flow model. Numerical analysis of vertical discharging of the water vapor in the liquid sodium pool was also performed by using the newly constructed droplet entrainment / transport model as well as the existing flow and chemical reaction model. The basic function of the droplet entrainment / transport model was discussed.

\section{Nomenclature}

$a \quad$ interfacial area density $(1 / \mathrm{m})$

$C_{D} \quad$ drag coefficient

$C_{p} \quad$ specific heat $(\mathrm{J} / \mathrm{kg} / \mathrm{K})$

$D_{m} \quad$ effective coefficient of diffusion $\left(\mathrm{m}^{2} / \mathrm{s}\right)$

D diameter (m)

$d_{0} \quad$ diameter of gas jet after underexpansion (m)

$\mathbf{F}^{d} \quad$ interfacial drag force $\left(\mathrm{kg} / \mathrm{m}^{2} / \mathrm{s}^{2}\right)$ 
$G^{d i f} \quad$ source term by species diffusion $\left(\mathrm{kg} / \mathrm{m}^{3} / \mathrm{s}\right)$

$G^{s f} \quad$ mass generation rate by surface reaction $\left(\mathrm{kg} / \mathrm{m}^{3} / \mathrm{s}\right)$

g gravity vector $\left(\mathrm{m} / \mathrm{s}^{2}\right)$

$H \quad$ coefficient of heat transfer $\left(\mathrm{W} / \mathrm{m}^{2} / \mathrm{K}\right)$

$h \quad$ enthalpy $(\mathrm{J} / \mathrm{kg})$

$i \quad$ latent heat $(\mathrm{J} / \mathrm{kg})$

Le Lewis number

$l \quad$ characteristic length $(\mathrm{m})$

$M_{W} \quad$ molecular weight $(\mathrm{g} / \mathrm{mol})$

$\dot{m}_{e} \quad$ droplet entrainment rate $\left(\mathrm{kg} / \mathrm{m}^{2} / \mathrm{s}\right)$

$p \quad$ pressure $(\mathrm{Pa})$

$Q^{\text {dif }} \quad$ source term by species diffusion $\left(\mathrm{W} / \mathrm{m}^{3}\right)$

$Q^{s f} \quad$ heat generation rate by surface reaction $\left(\mathrm{W} / \mathrm{m}^{3}\right)$

$R \quad$ universal gas constant $(\mathrm{J} / \mathrm{mol} / \mathrm{K})$

Re Reynolds number

$S^{e} \quad$ droplet entrainment rate $\left(\mathrm{kg} / \mathrm{m}^{3} / \mathrm{s}\right)$

$S^{d} \quad$ droplet deposition rate $\left(\mathrm{kg} / \mathrm{m}^{3} / \mathrm{s}\right)$

Sh Sherwood number

$T \quad$ temperature (K)

$T_{s} \quad$ saturation temperature $(\mathrm{K})$

u velocity vector $(\mathrm{m} / \mathrm{s})$

$u_{0} \quad$ velocity of gas jet after underexpansion $(\mathrm{m} / \mathrm{s})$

$Y \quad$ mass fraction

$z \quad$ coefficient of compressibility

\section{Greek symbols}

$\alpha \quad$ volume fraction

$\Phi$ dissipation function $\left(\mathrm{W} / \mathrm{m}^{3}\right)$

$\Gamma^{c} \quad$ condensation rate $\left(\mathrm{kg} / \mathrm{m}^{3} / \mathrm{s}\right)$

$\Gamma^{e} \quad$ evaporation rate $\left(\mathrm{kg} / \mathrm{m}^{3} / \mathrm{s}\right)$

$\gamma \quad$ specific heat ratio

$\gamma^{f f} \quad$ mass generation rate by surface reaction $\left(\mathrm{kg} / \mathrm{m}^{3} / \mathrm{s}\right)$

$\lambda$ thermal conductivity $(\mathrm{W} / \mathrm{m} / \mathrm{K})$

$\mu \quad$ viscosity (Pa s)

$\rho \quad$ density $\left(\mathrm{kg} / \mathrm{m}^{3}\right)$

$\rho_{0} \quad$ density of gas jet after underexpansion $\left(\mathrm{kg} / \mathrm{m}^{3}\right)$

$\sigma \quad$ surface tension $(\mathrm{N} / \mathrm{m})$

$\tau \quad$ viscous stress tensor $\left(\mathrm{kg} / \mathrm{m}^{2} / \mathrm{s}^{2}\right)$

$\omega \quad$ relaxation factor

\section{Superscript}

$m \quad$ iteration step

* critical state

\section{Subscript}

$c \quad$ continuous phase

d droplet phase

$g \quad$ gas phases

$j \quad$ species

$l \quad$ liquid phase

$0 \quad$ stagnation point 


\section{Numerical Methods}

\subsection{Governing Equations}

The multiphase flow under the tube failure accident is calculated by the multi-fluid model considering compressibility. The first phase is the multicomponent gas with water vapor, sodium hydroxide $(\mathrm{NaOH})$ aerosol, hydrogen, sodium vapor and $\mathrm{NaOH}$ vapor. The second phase is the liquid sodium. The liquid sodium is entrained at the gas-liquid interface (i.e., interface of gaseous jet) and the sodium droplets are transported in the gaseous flow. We considered the entrained droplets as a dispersed phase and added the governing equations of the droplet phase in the present work. Governing equations for the gas, liquid and droplet phase are as follows:

Equation of mass conservation

$$
\begin{aligned}
& \frac{\partial}{\partial t}\left(\alpha_{g} \rho_{g}\right)+\nabla \cdot\left(\alpha_{g} \rho_{g} \mathbf{u}_{g}\right)=\Gamma_{l}^{e}+\Gamma_{d}^{e}-\Gamma^{c}+G_{l}^{s f}+G_{d}^{s f}+G^{d i f} \\
& \frac{\partial}{\partial t}\left(\alpha_{l} \rho_{l}\right)+\nabla \cdot\left(\alpha_{l} \rho_{l} \mathbf{u}_{l}\right)=-\Gamma_{l}^{e}+\Gamma^{c}-G_{l}^{s f}-S^{e}+S^{d} \\
& \frac{\partial}{\partial t}\left(\alpha_{d} \rho_{d}\right)+\nabla \cdot\left(\alpha_{d} \rho_{d} \mathbf{u}_{d}\right)=-\Gamma_{d}^{e}-G_{d}^{s f}+S^{e}-S^{d}
\end{aligned}
$$

Equation of momentum conservation

$$
\begin{aligned}
& \frac{\partial}{\partial t}\left(\alpha_{g} \rho_{g} \mathbf{u}_{g}\right)+\nabla \cdot\left(\alpha_{g} \rho_{g} \mathbf{u}_{g} \mathbf{u}_{g}\right)=-\alpha_{g} \nabla p+\nabla \cdot\left(\alpha_{g} \boldsymbol{\tau}_{g}\right)+\alpha_{g} \rho_{g} \mathbf{g} \\
& +\mathbf{F}_{g l}^{d}+\mathbf{F}_{g d}^{d}+\Gamma_{l}^{e} \mathbf{u}_{l}+\Gamma_{d}^{e} \mathbf{u}_{d}-\Gamma^{c} \mathbf{u}_{g}+G_{l}^{s f} \mathbf{u}_{l}+G_{d}^{s f} \mathbf{u}_{d}+G^{d i f} \mathbf{u}_{g} \\
& \frac{\partial}{\partial t}\left(\alpha_{l} \rho_{l} \mathbf{u}_{l}\right)+\nabla \cdot\left(\alpha_{l} \rho_{l} \mathbf{u}_{l} \mathbf{u}_{l}\right)=-\alpha_{l} \nabla p+\nabla \cdot\left(\alpha_{l} \boldsymbol{\tau}_{l}\right)+\alpha_{l} \rho_{l} \mathbf{g} \\
& -\mathbf{F}_{g l}^{d}-\Gamma_{l}^{e} \mathbf{u}_{l}+\Gamma^{c} \mathbf{u}_{g}-G_{l}^{s f} \mathbf{u}_{l}-S^{e} \mathbf{u}_{l}+S^{d} \mathbf{u}_{d} \\
& \frac{\partial}{\partial t}\left(\alpha_{d} \rho_{d} \mathbf{u}_{d}\right)+\nabla \cdot\left(\alpha_{d} \rho_{d} \mathbf{u}_{d} \mathbf{u}_{d}\right)=-\alpha_{d} \nabla p+\alpha_{d} \rho_{d} \mathbf{g} \\
& -\mathbf{F}_{g d}^{d}-\Gamma_{d}^{e} \mathbf{u}_{d}-G_{d}^{s f} \mathbf{u}_{d}+S^{e} \mathbf{u}_{l}-S^{d} \mathbf{u}_{d}
\end{aligned}
$$

Equation of energy conservation

$$
\begin{aligned}
& \frac{\partial}{\partial t}\left(\alpha_{g} \rho_{g} h_{g}\right)+\nabla \cdot\left(\alpha_{g} \rho_{g} h_{g} \mathbf{u}_{g}\right)=\alpha_{g} \frac{D p}{D t}+\alpha_{g} \Phi_{g}+\nabla \cdot\left(\alpha_{g} \lambda_{g} \nabla T_{g}\right) \\
& +a_{g l} H_{g l}\left(T_{l}-T_{g}\right)+a_{g d} H_{g d}\left(T_{d}-T_{g}\right)+\Gamma_{l}^{e}\left(h_{l}+i_{l}\right)+\Gamma_{d}^{e}\left(h_{d}+i_{d}\right)-\Gamma^{c} h_{g y} \\
& +Q^{s f}+Q^{d i f} \\
& \frac{\partial}{\partial t}\left(\alpha_{l} \rho_{l} h_{l}\right)+\nabla \cdot\left(\alpha_{l} \rho_{l} h_{l} \mathbf{u}_{l}\right)=\alpha_{l} \frac{D p}{D t}+\alpha_{l} \Phi_{l}+\nabla \cdot\left(\alpha_{l} \lambda_{l} \nabla T_{l}\right) \\
& +a_{g l} H_{g l}\left(T_{g}-T_{l}\right)-\Gamma_{l}^{e}\left(h_{l}+i_{l}\right)+\Gamma^{c} h_{g y}-G_{l}^{s f} h_{l}-S^{e} h_{l}+S^{d} h_{d} \\
& \frac{\partial}{\partial t}\left(\alpha_{d} \rho_{d} h_{d}\right)+\nabla \cdot\left(\alpha_{d} \rho_{d} h_{d} \mathbf{u}_{d}\right)=\alpha_{d} \frac{D p}{D t} \\
& +a_{g d} H_{g d}\left(T_{g}-T_{d}\right)-\Gamma_{d}^{e}\left(h_{d}+i_{d}\right)-G_{d}^{s f} h_{d}+S^{e} h_{l}-S^{d} h_{d}
\end{aligned}
$$

where $\alpha$ is the volume fraction, $\rho$ the density, $\mathbf{u}$ the velocity vector, $\Gamma^{e}$ the evaporation rate, $\Gamma^{c}$ the condensation rate, $G^{s f}$ the mass generation rate by surface reaction, $G^{\text {dif }}$ the source term by species diffusion, $S^{e}$ the droplet entrainment rate, $S^{d}$ the droplet deposition rate, $p$ the pressure, $\tau$ the viscous stress tensor, $\mathbf{g}$ the gravity vector, $\mathbf{F}^{d}$ the interfacial drag force, $h$ the enthalpy, $\Phi$ the dissipation function, $\lambda$ the thermal conductivity, $T$ the temperature, $a$ the 
interfacial area density, $H$ the coefficient of heat transfer, $i$ the latent heat, $h_{g y}$ the enthalpy of the species, $Q^{\text {sf }}$ the heat generation rate by surface reaction and $Q^{d i f}$ the source term by species diffusion. Subscript $g$ stands for the gas phase, $l$ the liquid phase and $d$ the droplet phase. Numerical models to calculate the source term will be described later.

The equation of state is given by

$$
p=z \frac{\rho_{g}}{M_{W}} R T_{g}
$$

where $z$ is the coefficient of compressibility, $M_{W}$ the molecular weight and $R$ the universal gas constant. The ideal gas approximation $(z=1)$ gives certain errors under the high-pressure condition such as the water vapor in the SG. In our method, the coefficient of compressibility is calculated from the modified Benedict-Webb-Rubin equation ${ }^{(7)}$ which gives the actual gas-phase density. The density of the liquid phase is estimated from the polynomial equation of the temperature and pressure.

Transport of the species $j$ is calculated from the advection-diffusion equation:

$$
\frac{\partial}{\partial t}\left(\alpha_{g} \rho_{g} Y_{j}\right)+\nabla \cdot\left(\alpha_{g} \rho_{g} Y_{j} \mathbf{u}_{g}\right)=\nabla \cdot\left(\alpha_{g} \rho_{g} D_{m j} \nabla Y_{j}\right)+\Gamma_{j}^{e}-\Gamma_{j}^{c}+\gamma_{j}^{s f}
$$

where $Y$ is the mass fraction, $D_{m}$ the effective coefficient of diffusion and $\gamma^{s f}$ the source term by surface reaction.

The governing equations are solved by the semi-implicit method. The time-derivative terms in Eqs. (1) to (9) and (11) were approximated by the forward difference scheme. Only the convective and diffusion terms were estimated explicitly. The second-order TVD scheme was chosen for the convective terms in the equation of momentum conservation. The convective terms in other equations were discretized by the first-order upwind difference scheme. The second-order central difference scheme was applied to the viscous, heat conduction and diffusion terms.

\subsection{Chemical Reaction Model}

In our previous studies ${ }^{(1-3)}$, we developed a numerical model for the chemical reaction at the interface between water vapor and liquid sodium. The model is called a surface reaction model. The surface reaction model is outlined below.

The following chemical reaction is dominant between water vapor and liquid sodium $^{(1-3)}$ (see Fig. 2).

$$
\mathrm{Na}+\mathrm{H}_{2} \mathrm{O} \rightarrow \mathrm{NaOH}+\frac{1}{2} \mathrm{H}_{2}
$$

The surface reaction model calculates mass generation rate in the above reaction, which is the source term appearing in the governing equation. The model is based on the assumption of infinite reaction rate. In other words, progress of the chemical reaction at the gas-liquid interface is limited by the mass flow rate of the reactant gas toward the interface. The mass flow rate of the reactant gas $j$ is written as

$$
\gamma_{j}^{s f}=S h \frac{D_{m j}}{l} \rho_{g} Y_{j} a
$$

where $S h$ is the Sherwood number $(S h=k l / D), k$ the coefficient of mass transfer, $l$ the 


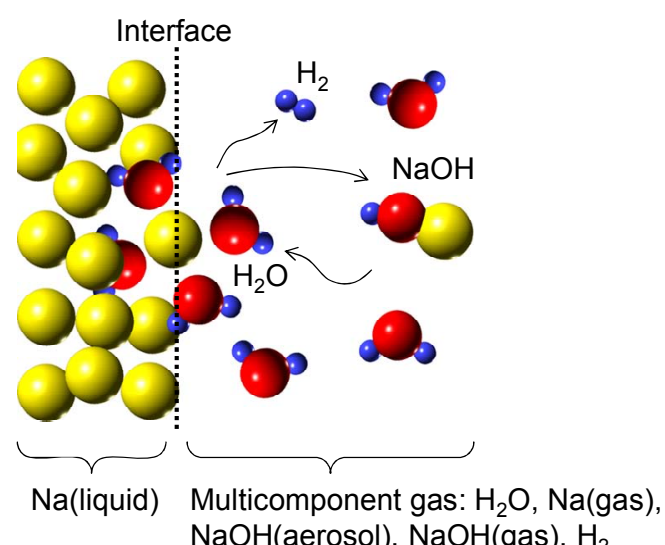

Fig. 2 Chemical Reaction at Interface between Water Vapor and Liquid Sodium

characteristic length. Equation (13) includes some unknown parameters. Considering an analogy between the heat and mass transfer, Eq. (13) is rewritten as

$$
\gamma_{j}^{s f}=-L e^{b-1} \frac{H_{g l}}{C_{p g}} Y_{j} a
$$

where $L e$ is the Lewis number $(L e=\alpha / D), \alpha$ the coefficient of thermal diffusivity, $b$ the empirical constant and $C_{p g}$ the gas-phase specific heat. The Lewis number is calculated according to its definition. The experimental heat transfer correlations for turbulent flow indicate that the empirical constant $b$ becomes less than unity. Furthermore, there is the knowledge that $b$ is not so effective on the reacting zone ${ }^{(8)}$. Hence, $b$ was set to 0 in the present analysis. Tanabe et al. ${ }^{(9)}$ conducted the experiment on water vapor leakage into sodium and reported that the coefficient of heat transfer on the surface of a heat transfer tube in a reacting zone was approximately $10000 \mathrm{~W} / \mathrm{m}^{2} / \mathrm{K}$. Heat transfer on the gas-liquid interface seems to be similar to that on the tubes because the liquid-phase velocity is much smaller than the gas-phase velocity. For this reason, we applied the coefficient of heat transfer measured on the tube to that on the gas-liquid interface, $H_{g l}$. The interfacial area density $a$ is given by a Nigmatulin model ${ }^{(10)}$.

We have supposed that the reaction products, $\mathrm{NaOH}$ and hydrogen, move to the gas phase. Therefore, the SERAPHIM program deals with the multicomponent gas phase.

\subsection{Evaporation / Condensation Rate}

The evaporation / condensation rate is written as ${ }^{(10,11)}$ :

$$
\begin{gathered}
\Gamma^{e}= \begin{cases}\lambda_{e} a \rho_{l} \alpha_{g} \sqrt{\frac{R}{M_{W}}}\left(1-\alpha_{g}\right) \frac{\left(T_{l}-T_{s}\right)}{\sqrt{T_{s}}} & T_{l} \geq T_{s} \\
0 & T_{l}<T_{s}\end{cases} \\
\Gamma^{c}= \begin{cases}0 & T_{g}>T_{s} \\
\lambda_{c} a \rho_{g}\left(1-\alpha_{g}\right) \sqrt{\frac{R}{M_{W}}} \alpha_{g} \frac{\left(T_{g}-T_{s}\right)}{\sqrt{T_{s}}} & T_{g} \leq T_{s}\end{cases}
\end{gathered}
$$

where $\lambda_{e}$ and $\lambda_{c}$ are the empirical constants and $T_{s}$ the saturation temperature. Based on the past researches, the empirical constant $\lambda_{e}$ and $\lambda_{c}$ were determined to be 0.1. Takata and Yamaguchi ${ }^{(1)}$ analyzed Edwards pipe blowdown problem by using the SERAPHIM program and validated applicability of the above model. 


\subsection{Interfacial Drag Force}

The gas-liquid interfacial drag force was calculated from the assumption mentioned below. The applicability of the present model has been investigated through the analyses of the basic experiments ${ }^{(12)}$. The analysis of the argon gas jet in the rod-inserted water pool is also performed as one of the validations of the multi-fluid model and the interfacial drag force model.

The gas-liquid interfacial drag force model used in the present work is based on a high mixing volume flow regime map ${ }^{(13)}$. As with the map, a flow pattern was assumed as follows: bubbly flow for $\alpha<0.5$, droplet flow for $\alpha>0.95$ and transition region between them. The gas-liquid interfacial drag force term in Eq. (5) is given by

$$
\mathbf{F}_{g l}^{d}=-\frac{3}{4} \frac{1}{D} \alpha \rho_{c} C_{D, g l}\left|\mathbf{u}_{g}-\mathbf{u}_{l}\right|\left(\mathbf{u}_{g}-\mathbf{u}_{l}\right)
$$

where $D$ is the diameter of the bubble or droplet and $C_{D}$ the drag coefficient. Subscript $c$ stands for the continuous phase. We estimated the drag coefficient between the gas and liquid phase from the well-known relationship ${ }^{(14)}$ :

$$
C_{D, g l}= \begin{cases}\frac{24}{\mathrm{Re}} & \mathrm{Re}<1 \\ \frac{24}{\operatorname{Re}}\left(1+0.15 \mathrm{Re}^{0.687}\right) & 0.1 \leq \mathrm{Re}<1000 \\ 0.44 & \mathrm{Re} \geq 1000\end{cases}
$$

where

$$
\operatorname{Re}=\frac{\left|\mathbf{u}_{g}-\mathbf{u}_{l}\right| D \rho_{c}}{\mu_{c}}
$$

An interfacial drag force in the transition region was presumed to be void-fraction-weighted average of the two drag forces. The drag force between the gas and entrained droplet $\mathbf{F}_{g d}^{d}$ in Eq. (6) is also given by Eq. (17). Walsh proposed the following correlation for the drag coefficient of the moving solid particles in a supersonic gaseous flow ${ }^{(15)}$.

$$
C_{D}=\frac{24}{\operatorname{Re}}\left(1+0.15 \mathrm{Re}^{0.687}\right)\left[1+\exp \left(-\frac{0.427}{M_{r}^{4.63}}-\frac{3}{\mathrm{Re}^{0.88}}\right)\right]
$$

where

$$
M_{r}=\frac{\left|\mathbf{u}_{g}-\mathbf{u}_{d}\right|}{\sqrt{\gamma R T_{g}}}
$$

and $\gamma$ is the specific heat ratio. Equation (20) was applied to the drag coefficient of the entrained droplet $C_{D, g d}$.

\subsection{Droplet Entrainment Rate and Diameter}

The numerical model for the sodium droplet entrainment at the gas-liquid interface was newly constructed in this study. To estimate the droplet entrainment rate, which is the 
source term in the governing equations, we applied the following Ricou-Spalding equation $^{(16)}$

$$
\dot{m}_{e}=E_{0}\left(\rho_{g} \rho_{l}\right)^{1 / 2}\left|\mathbf{u}_{g}-\mathbf{u}_{l}\right|
$$

where $\dot{m}_{e}$ is the droplet entrainment rate and $E_{0}$ the empirical constant. Liquid entrainment by gas jets has been investigated in several studies ${ }^{(17-20)}$. These studies all demonstrated that the entrainment rate is represented well by the equation in the same functional form as Eq. (22). Epstein et al. ${ }^{(21)}$ investigated behavior of the steam jet in liquid sodium analytically by the one-dimensional theoretical model. They used Eq. (22) with $E_{0}=0.08$ and reproduced the temperature profile successfully. As with the research by Epstein et al., the empirical constant $E_{0}$ was assumed to be 0.08 in our model. The droplet entrainment rate $S^{e}$ appearing in the governing equations was obtained by multiplying the interfacial area density to Eq. (22). Thus, the droplet phase is generated at the region in which the interfacial area density is high. The interfacial area density reaches its peak at $\alpha=0.5$ in our model.

Epstein et al. ${ }^{(22)}$ measured the diameter of the droplet entrained by the gas jet and derived the following correlation describing Sauter mean diameter (SMD).

$$
\frac{\mathrm{SMD}}{d_{0}}=0.39\left(\frac{\sigma}{u_{0}^{2} \rho_{0} d_{0}}\right)^{0.4}
$$

where $d_{0}, u_{0}$ and $\rho_{0}$ are the diameter, velocity and density of the gas jet after underexpansion and $\sigma$ the surface tension. To estimate the drag force and the interfacial area density between the gas and entrained droplet, we calculate the droplet diameter from Eq. (23)

The entrained sodium droplet will also react with the water vapor. The above-mentioned surface reaction model was applied to the chemical reaction of the droplet phase.

\subsection{HSMAC Method for Compressible Multiphase Flow}

The set of the governing equations is solved by the HSMAC (Highly Simplified Marker And Cell) method modified for compressible multiphase flows. The HSMAC method for incompressible single-phase flows was developed by Hirt and $\operatorname{Cook}^{(23)}$. In this method, the pressure and the velocity are corrected so as to satisfy continuity by using the Newton-Raphson method. The HSMAC method is very useful for parallel computation because there is no need to solve a simultaneous linear equation. Extension of the HSMAC method to compressible multiphase flows is described here.

The recurrence equation for the pressure correction is written as

$$
p^{m+1}=p^{m}+\delta p=p^{m}-D^{m} /\left(\frac{\partial D}{\partial p}\right)^{m}
$$

where superscript $m$ stands for the iteration step. The variable $D$ approaches to 0 from iterative calculation. In the case of multiphase flows, similarly to the method proposed by Matsumoto and Murai ${ }^{(24)}$, we define the variable $D$ by

$$
D=-\sum_{k} \frac{\partial \alpha_{k}}{\partial t}
$$

Substituting Eqs. (1) to (3) into Eq. (25), $D$ is rewritten as 


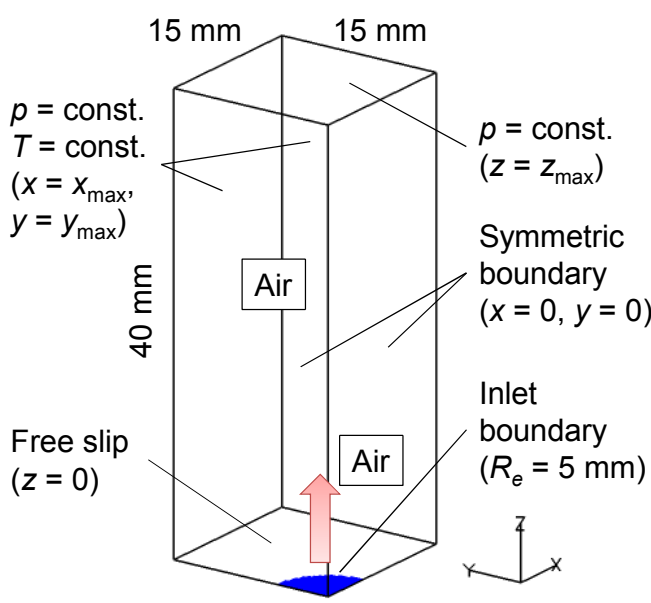

Fig. 3 Computational Domain of Air Jet in Air

$$
D=\sum_{k}\left\{\frac{\alpha_{k}}{\rho_{k}} \frac{\partial \rho_{k}}{\partial t}+\frac{1}{\rho_{k}} \nabla \cdot\left(\alpha_{k} \rho_{k} \mathbf{u}_{k}\right)\right\}
$$

From Eqs. (4) to (6), (10), (24) and (26), we can derive a final form of pressure correction:

$$
\delta p=-\omega \frac{D^{m}+\sum_{k}\left\{\frac{\alpha_{k}^{n}}{\rho_{k}^{n}}\left(\frac{\partial \rho_{k}}{\partial T_{k}} \frac{\delta T_{k}}{\Delta t}+\frac{\partial \rho_{k}}{\partial M} \frac{\delta M}{\Delta t}\right)\right\}}{\sum_{k}\left[\frac{\alpha_{k}^{n}}{\rho_{k}^{n}}\left\{\frac{\partial \rho_{k}}{\partial p} \frac{1}{\Delta t}+2 \Delta t\left(\frac{1}{\Delta x^{2}+\Delta y^{2}+\Delta z^{2}}\right)\right\}\right]}
$$

where $\omega$ is the relaxation factor. The $m+1$-th pressure is calculated from Eqs. (24) and (27). The velocity is updated after the pressure correction. When the residual calculated from Eq. (25) converges, we move to the next step calculation. For stable calculation, the Courant number must be lower than about 0.3 .

\section{Results and Discussion}

\subsection{Analysis of Underexpanded Air Jet in Air}

Numerical analysis of the underexpanded air jet in the air was performed for basic validation of the governing equations and the solution method considering compressibility. The analysis conditions are described below. We consider the three-dimensional rectangular region $\left(x_{\max }=15 \mathrm{~mm}, y_{\max }=15 \mathrm{~mm}, z_{\max }=40 \mathrm{~mm}\right.$ ) shown in Fig. 3. The region is filled with the air at the temperature of $20^{\circ} \mathrm{C}$ and the pressure of $0.1 \mathrm{MPa}$. The pressurized air goes into the region from the inlet boundary simulating the nozzle exit with $5 \mathrm{~mm}$ radius. The stagnation pressure $p_{0}$ and temperature $T_{0}$ of the air was set to $0.617 \mathrm{MPa}$ and $20{ }^{\circ} \mathrm{C}$ as with the experimental condition by Kuehner et al. ${ }^{(4)}$ or Woodmansee et al. ${ }^{(5)}$. The air at the inlet boundary is in the critical state. Assuming an isentropic flow, the physical quantities at the nozzle exit are given by the well-known relationships:

$$
\begin{aligned}
& u^{*}=\sqrt{\frac{2 \gamma}{\gamma+1} \frac{p_{0}}{\rho_{0}}} \\
& p^{*}=p_{0}\left(\frac{2}{\gamma+1}\right)^{\frac{\gamma}{\gamma-1}}
\end{aligned}
$$




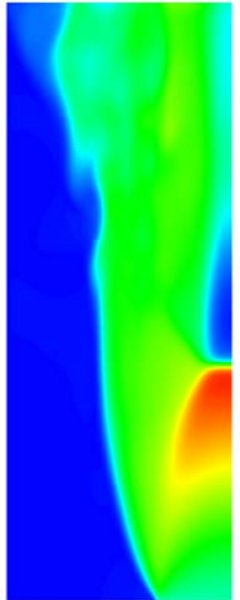

Mach number

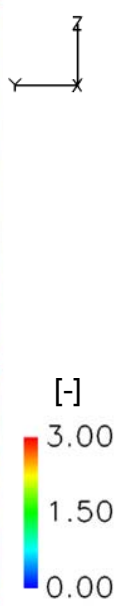

$[-]$

3.00
1.50
0.00

(1)

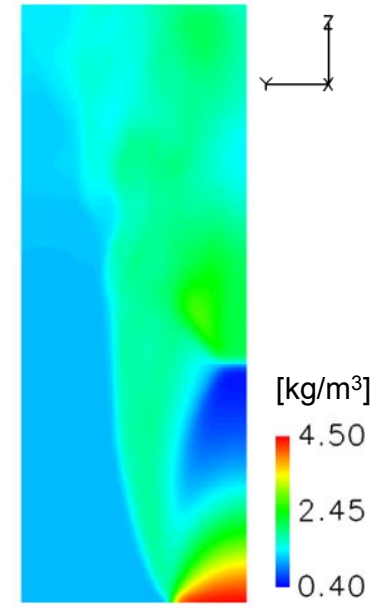

Density

Fig. 4 Numerical Results of Air Jet in Air

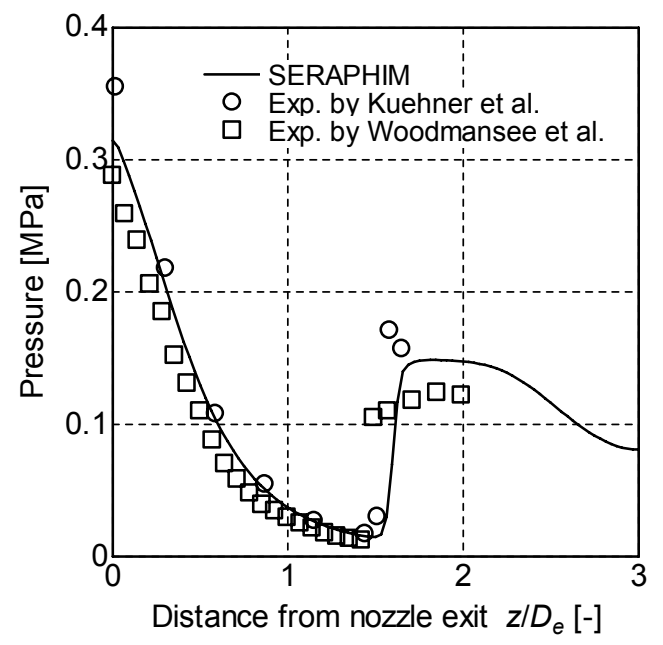

Fig. 5 Profile of Pressure along Centerline of Jet

$$
\begin{aligned}
& T^{*}=T_{0} \frac{2}{\gamma+1} \\
& \rho^{*}=\rho_{0}\left(\frac{2}{\gamma+1}\right)^{\frac{1}{\gamma-1}}
\end{aligned}
$$

where superscript * stands for the critical state and subscript 0 stands for the stagnation state. In this analysis, the velocity at the inlet boundary becomes approximately $314 \mathrm{~m} / \mathrm{s}$ (sound speed). The pressure at the surfaces of $x=x_{\max }, y=y_{\max }$ and $z=z_{\max }$ was assumed to be constant at $0.1 \mathrm{MPa}$ (ambient pressure). While the temperature at the surfaces of $x=x_{\max }$ and $y=y_{\max }$ was assumed to be constant at $20^{\circ} \mathrm{C}$ (ambient temperature), the temperature at $z=z_{\max }$ was extrapolated from the value inside the region because the air goes out from this boundary. The symmetric condition was applied to the surface of $x=0$ and $y=0$. The computational domain was divided into the equally-spaced mesh with a cell $0.3125 \mathrm{~mm}$ on a side.

An underexpanded jet appears when an ambient pressure is lower than a critical pressure $p^{*}$. In the case of $p_{0}=0.617 \mathrm{MPa}$, the critical pressure $p^{*}$ is about $0.32 \mathrm{MPa}$ and this is higher than the ambient pressure $(0.1 \mathrm{MPa})$. Figure 4 shows the instantaneous 


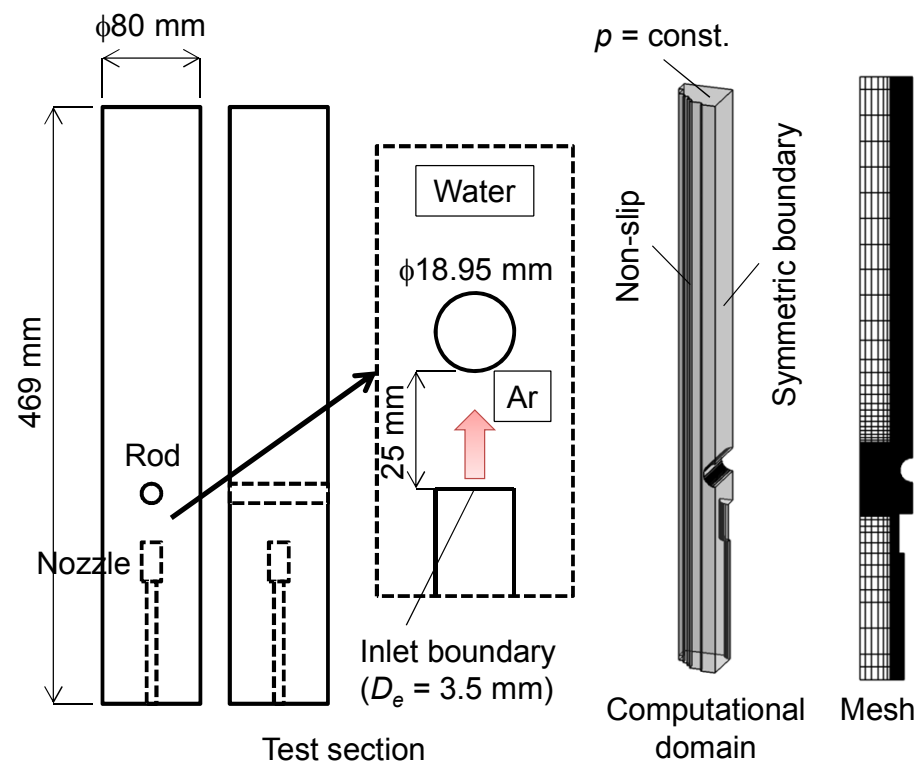

Fig. 6 Computational Domain of Argon Gas Jet in Water

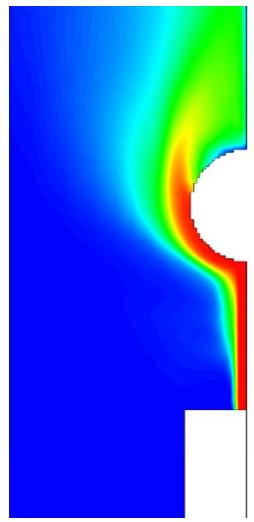

(a) Void fraction

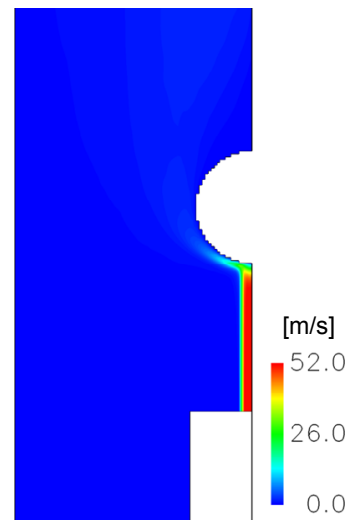

(b) Gas-phase velocity

Fig. 7 Numerical Results of Argon Gas Jet in Water

distributions of the Mach number and density. It can be seen that the air expands after exiting the nozzle and the shock wave called Mach disk appears in the jet. The numerical result shown in Fig. 4 represents the well-known structure of the underexpanded jet. Figure 5 shows the profile of the time-averaged pressure along the centerline of the jet. The pressure decreases with underexpansion and varies sharply at the Mach disk $\left(z / D_{e} \approx 1.5\right)$. The calculated pressure shows good agreement with the experimental data by Kuehner et al. or by Woodmansee et al.

\subsection{Analysis of Argon Gas Jet in Water Pool}

The computational domain for analysis of the argon gas jet into the water pool shown in Fig. 6 simulates the test section in the experiment by Kudo et al. ${ }^{(6)}$. The length of the computational domain is $469 \mathrm{~mm}$ and the inner diameter $80 \mathrm{~mm}$. The domain was initially filled with the water at temperature of $20{ }^{\circ} \mathrm{C}$ and hydrostatic pressure. There is the gas supply nozzle and the inlet boundary at $129 \mathrm{~mm}$ above the bottom surface. The area of the inlet boundary was set to be equal to a cross-section area of the $3.5 \mathrm{~mm}$ diameter hole. The rod with $18.95 \mathrm{~mm}$ diameter is installed above the nozzle. The distance between the inlet boundary and the bottom edge of the rod is $25 \mathrm{~mm}$. The air goes into the water pool vertically from the inlet boundary with the velocity of $51.9 \mathrm{~m} / \mathrm{s}$ and the void fraction of 1 , 


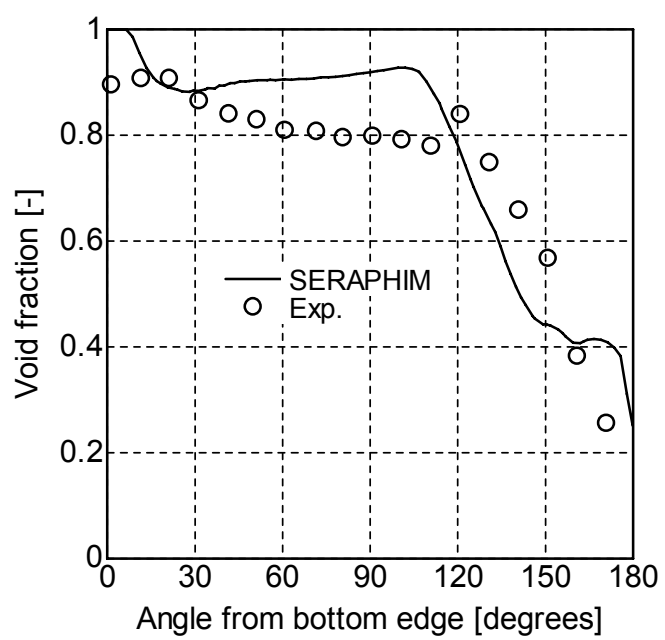

Fig. 8 Profile of Void Fraction along Rod Surface

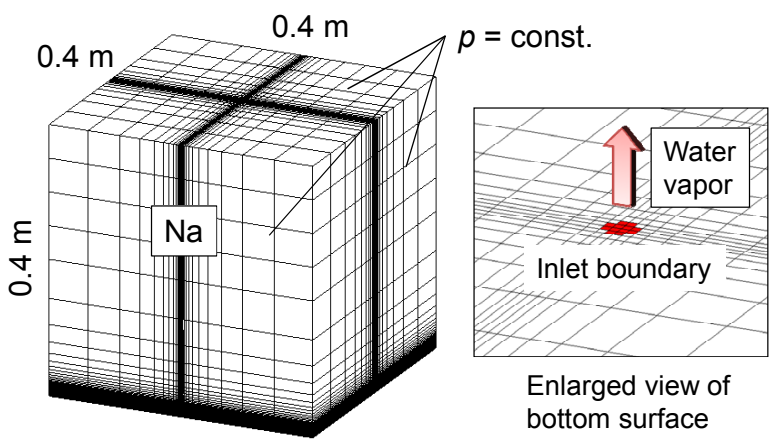

Fig. 9 Computational Domain of Water Vapor Jet in Sodium

and then impinges the rod. The pressure at the top surface was set to be constant at $0.1 \mathrm{MPa}$. The gas phase can goes out from the top surface. A non-slip condition was applied to the side surface of the vessel. Analysis mesh was constructed with unequally-spaced cells.

Figure 7 shows the time-averaged (1 second) distributions of the void fraction and gas-phase velocity on a vertical plane intersecting with the center of the inlet boundary. The numerical result shows that the air goes upward around the rod. The void fraction in the upper side of the rod is lower than that in the underside. This tendency can be found even in Fig. 8. This figure shows the profile of the void fraction on the cylindrical surface which is $2 \mathrm{~mm}$ distant from a rod surface. The horizontal axis stands for the angle from the bottom edge of the rod. The measured void fraction gradually decreases in the region less than 120 degrees. There is a certain level of the difference between the numerical and the experimental results. The numerical results may depend on a method to estimate a gas-liquid interfacial drag force or application of the droplet entrainment / transport model. A sensitivity of the numerical models must be investigated to improve the prediction accuracy in further research. Experimental result shows that the void fraction steeply decreases in the region greater than 120 degrees. This tendency appeared also in the numerical results. The investigation into the model capability for the other velocity conditions are expected in the future research.

\subsection{Analysis of Water Vapor Jet in Sodium Pool}

The SERAPHIM program which incorporates the droplet entrainment / transport model was tested through the analysis of vertical discharging of the water vapor in the liquid sodium pool. In this test analysis, to investigate basic applicability of the chemical reaction 


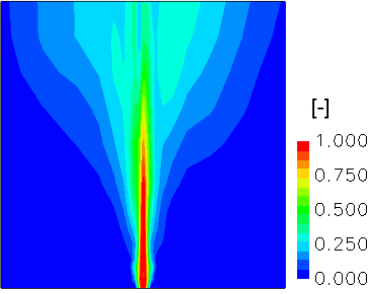

(a) Void fraction

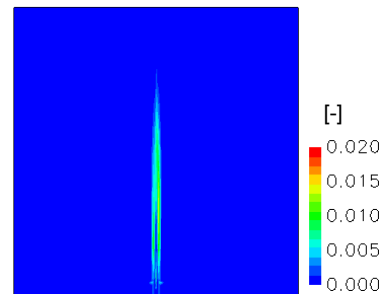

(b) Volume fraction of droplet

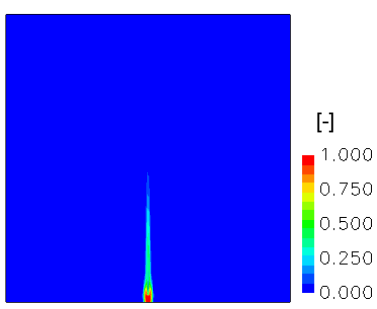

(c) Volume fraction of $\mathrm{H}_{2} \mathrm{O}$

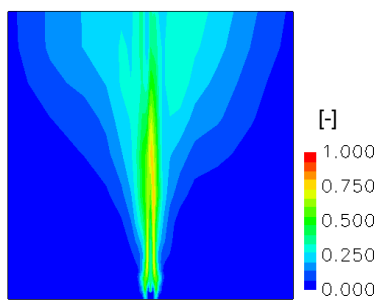

(d) Volume fraction of $\mathrm{H}_{2}$

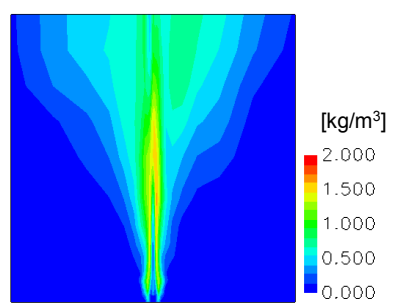

(e) Volume fraction of $\mathrm{NaOH}$
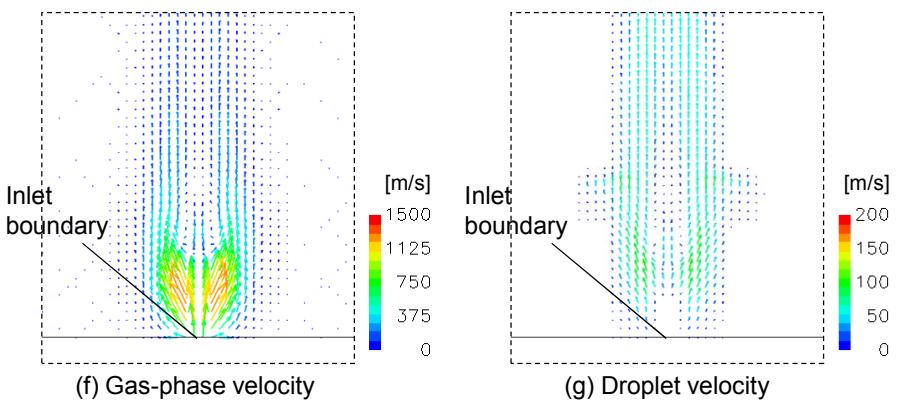

(g) Droplet velocity

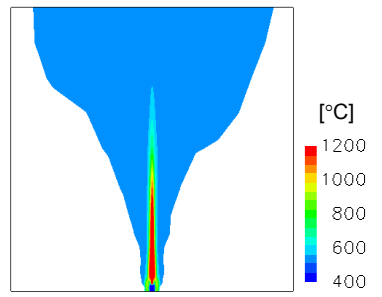

(h) Gas-phase temperature

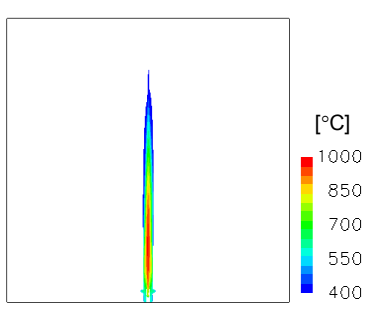

(i) Droplet temperature

Fig. 10 Numerical Results of Water Vapor Jet in Sodium

model and the droplet entrainment / transport model, the simple-shaped computational domain shown in Fig. 9 was used. Both the width and the height of the domain is $0.4 \mathrm{~m}$. The domain was initially filled with the liquid sodium at the temperature of $520^{\circ} \mathrm{C}$. There is an inlet boundary at the center of the bottom surface. The area of the inlet boundary was set to be equal to the cross-section area of the $2 \mathrm{~mm}$ diameter hole. The water vapor goes into the pool vertically and reacts with the sodium. The flow of the water vapor becomes a critical state at the nozzle exit (i.e., inlet boundary). As mentioned above, the physical quantities were decided from the assumption of isentropic flow. The actual pressure and temperature inside the heat transfer tube $\left(p=17.3 \mathrm{MPa}, T=520^{\circ} \mathrm{C}\right)$ were regarded as a stagnation condition. In this case, the velocity at the inlet boundary becomes approximately $618 \mathrm{~m} / \mathrm{s}$ (sound speed). The gas, liquid and entrained droplet flow out from the top surface. The pressure on the top surface was assumed to be constant at $0.2 \mathrm{MPa}$. The pressure and temperature of the liquid sodium is same as the condition in the SG of the commercial fast reactor. From this analysis condition, Eq. (23) gives $67 \mu \mathrm{m}$ as SMD of the droplet.

Figure 10 shows the time-averaged ( 0.2 seconds $)$ distributions on a vertical plane intersecting with the center of the inlet boundary. As can be seen from Figs. 10(a) and (b), the droplet appears in the gas-phase region. This indicates that the newly constructed numerical model has a basic function to calculate the droplet entrainment and transport. In the future work, the prediction accuracy of the model is expected to be investigated. As 
shown in Fig. 10(c), the water vapor disappears by the chemical reaction with increasing distance from the inlet boundary. Instead, the hydrogen and the $\mathrm{NaOH}$ become a main species of the gas phase (Figs. 10(d) and (e)). The gas-phase velocity became locally higher than the inlet velocity and reached up to about $1500 \mathrm{~m} / \mathrm{s}$ (Fig. 10(f)). This implies occurrence of underexpansion of the water vapor jet. The maximum droplet velocity became about $160 \mathrm{~m} / \mathrm{s}$ (Fig. $10(\mathrm{~g})$ ). William et al. ${ }^{(25)}$ carried out the experiment on LDIE and clarified the effect of the impingement velocity of the droplet on the erosion rate. From the results obtained from this experiment, it is shown that the calculated liquid droplet velocity exceeds the occurrence condition of LDIE. The gas-phase temperature went up to about $1250{ }^{\circ} \mathrm{C}$ by the chemical reaction (Fig. 10(h)). Kurihara et al. ${ }^{(26)}$ measured the temperature distribution in the experiment on discharging of the water vapor in the liquid sodium pool in which the heat transfer tube bundle exists. The conditions of the pressure and temperature of their experiment are same as that in the present analysis. They reported that the maximum temperature went over $1200{ }^{\circ} \mathrm{C}$. This demonstrates that the peak temperature in the actual condition can be predicted by the use of the proposed surface reaction model.

\section{Conclusions}

Applicability of the multiphase flow model, solution method, chemical reaction model and newly constructed droplet entrainment / transport model was investigated. First, it was demonstrated that the governing equations and the HSMAC method considering compressibility could represent underexpanded jets, which is one of the most basic phenomena under tube failure accident. In the analysis of the argon gas jet in the rod-inserted water pool, the multiphase flow model gave good agreement with the tendency of the measured void fraction distribution. Numerical analysis of vertical discharging of the water vapor in the liquid sodium pool was performed. The peak temperature under the actual condition of the tube failure accident was reproduced by the use of the proposed surface reaction model. The newly constructed numerical model calculated the droplet entrainment and its transport successfully. Incorporation of the droplet entrainment / transport model made it possible to evaluate the environment of LDIE.

\section{Acknowledgments}

This work was partially supported by a study of "Development of Multi-Physics Evaluation System on Failure Propagation of Heat Transfer Tubes in the Steam Generator of Fast Breeder Reactor" entrusted to "Japan Atomic Energy Agency (JAEA)" by the Ministry of Education, Culture, Sports, Science and Technology of Japan (MEXT).

\section{References}

(1) Takata, T., and Yamaguchi, A., Numerical Approach to the Safety Evaluation of Sodium-Water Reaction, Journal of Nuclear Science and Technology, Vol.40, No.10(2003), pp.708-718.

(2) Takata, T., Yamaguchi, A., Fukuzawa, K., and Matsubara, K., Numerical Methodology of Sodium-Water Reaction with Multiphase Flow Analysis, Nuclear Science and Engineering, Vol.150(2005), pp.221-236.

(3) Takata, T., Yamaguchi, A., Uchibori, A., and Ohshima, H., Computational Methodology of Sodium-Water Reaction Phenomenon in Steam Generator of Sodium-Cooled Fast Reactor, Journal of Nuclear Science and Technology, Vol.46, No.6(2009), pp.613-623. 
(4) Kuehner, J. P., Dutton, J. C., and Rucht, R. P., High-Resolution $\mathrm{N}_{2}$ CARS Measurements of Pressure, Temperature, and Density Using a Modeless Dye Laser, AIAA, 2002-2915 (2002).

(5) Woodmansee, M. A., Experimental Measurements of Pressure, Temperature, and Density Using High-Resolution $\mathrm{N}_{2}$ Coherent Anti-Stokes Raman Scattering, Ph.D. thesis, University of Illinois at Urbana-Champaign, Urbana, IL (1999).

(6) Kudoh, H., Zhao, D., Sugiyama, K., Narabayashi, T., Ohshima, H., and Kurihara, A., Void Fraction Distributions of Inert Gas Jets across a Single Cylinder with Non-Wetting Surface in Liquid Sodium, Journal of Nuclear Science and Technology, Vol.49, No.12(2012), pp.1175-1185.

(7) Benedict, M., Webb, G. B., Rubin, L. C., An Empirical Equation for Thermodynamic Properties of Light Hydro-Carbons and Their Mixtures, Methane, Ethane, Propane and N-butane. Journal of Chemical Physics, Vol.8(1940), pp.334-345.

(8) Takata, T., Yamaguchi, A., and Watanabe, A., Modification of Multi-Dimensional Sodium-Water Reaction Analysis Code: SERAPHIM and Sensitivity Analyses on the Early Stage of Leakage, JNC TN9400 2003-024 (2003), Japan Nuclear Cycle Development Institute. [in Japanese]

(9) Tanabe, H., Wada, Y., Hamada, H., Miyagawa, A., and Hiroi, H., The Development and Application of Overheating Failure Model of FBR Steam Generator Tubes, PNC TN9410 98-029 (1976), Power Reactor and Nuclear Fuel Development Corporation. [in Japanese]

(10) Rivard, W. C., and Torrey, M. D., Numerical Calculation of Flashing from Long Pipes Using a Two-fluid Model, LA-NUREG-6330-MS (1976), Los Alamos Scientific Laboratory.

(11) Liles, D. R., and Reed, Wm. H., A Semi-implicit Method for Two-phase Fluid Dynamics, Journal of Computational Physics, Vol.26(1978), pp.390-407.

(12) Uchibori, A., Watanabe, A., and Ohshima, H., Numerical Analysis of Supersonic Gas Jets into Liquid Pools with or without Chemical Reaction Using the SERAPHIM Program," Nuclear Engineering and Design, Vol.249(2012), pp.35-40.

(13) The RELAP5 Code Development Team, RELAP5/MOD3 Code Manual, Volume I: Code Structure, System Models, and Solution Methods, INEL-95/0174 (1995), Idaho National Engineering Laboratory.

(14) W. C. Hinds, Aerosol Technology: Properties, Behavior, and Measurement of Airborne Particles, (1999), John Wiley \& Sons, Inc.

(15) Walsh, M. J., Influence of Particle Drag Coefficient on Particle Motion in High-speed Flow with Typical Laser Velocimeter Applications, NASA TN D-8120 (1976), National Aeronautics and Space Administration.

(16) Ricou, F. P., and Spalding, D. B., Measurements of Entrainment by Axisymmetrical Turbulent Jets, Journal of Fluid Mechanics, Vol.11(1961), pp.21-32.

(17) Bell, R. B., Boyce, E., and Collier, J. G., The Structure of a Submerged Impinging Gas Jet, Journal of the British Nuclear Energy Society, Vol.11(1972), pp.183-193.

(18) Carreau, J. L., Loukarfi, L., Gbahoue, L., Hobbes, P., and Roger, F., Hydrodynamics of an Axisymmetric, Submerged Non-reactive Gas Jet-measurement of Entrainment Contribution to the Wastage Modeling, Proceedings of the 20th Intersociety Energy Conversion Engineering Conference, (1985), pp.1.688-1.695.

(19) Loth, E., and Faeth, G. M., Structure of Underexpanded Round Air Jets Submerged in Water, International Journal of Multiphase Flow, Vol.15(1989), pp.589-603.

(20) Loth, E., and Faeth, G. M., Structure of Plane Underexpanded Air Jets into Water, AIChE Journal, Vol.36(1990), pp.818-826. 
(21) Epstein, M., Fauske, H. K., Yoshioka, N., Tashimo, M., Sakaba, H., and Kotake, S., Analytical Model for Peak Temperature within a Sodium-Water Reaction Jet, Journal of Nuclear Science and Technology. Vol.43, No.1(2006), pp.43-54.

(22) Epstein, M., Fauske, H. K., Yoshioka, N., Establishment of Analytical Model for Peak Temperature within a Sodium-Water Reaction Jet, (II) Mean Droplet Size in a Submerged Gas Jet, Journal of Nuclear Science and Technology. Vol.42, No.11(2005), pp.961-969.

(23) Hirt, C. W., and Cook, J. L., Calculating Three-Dimensional Flows around Structures and over Rough Terrain, Journal of Computational Physics, Vol.10(1972), pp.324-340.

(24) Matsumoto, Y., and Murai, Y., Numerical Simulation of Bubble Plume in a Tank with Free Surface, Transactions of the Japan Society of Mechanical Engineering, Series B, Vol.61, No.588(1995), pp.2818-2825. [in Japanese]

(25) William, K. C. W., Higashi, Y., Narabayashi, T., Tsuji, M., Ohshima, H., Kurihara, A., Study on Steam Generator Tube Wastage (9) Evaluation of LDI Test by Using High Speed Rotating Disc, Proceedings 2011 Fall Meeting of the Atomic Energy Society of Japan, P12(2012). [in Japanese]

(26) Kurihara, A., Shimoyama, K., Hamada, H., and Ohshima, H., Research on Sodium-Water Reaction Phenomena in Steam Generator of Sodium-Cooled Commercial Fast Reactor (2) Sodium-Water Reaction Experiment under Intermediate Water Leak Condition, Proceedings of the 2011 Annual Meeting of the Atomic Energy Society of Japan, H21(2011). [in Japanese] 